Ágora Rev. Cient. 2018; 05(02):e8

\title{
Saberes y prácticas de la persona con diabetes tipo 2: Implicancias para el cuidado de enfermería
}

\author{
Knowledge and practices of person with type 2 diabetes: Implications for nursing care \\ Gaby Sonia Chávez Zegarra ${ }^{1}$
}

\section{RESUMEN}

Objetivos: Describir saberes de la persona con diabetes tipo 2 (DM2) acerca de la enfermedad y cuidados requeridos, así como analizar prácticas de cuidado que realiza de acuerdo a sus saberes. Finalmente, discutir las implicancias de conocer sus saberes y prácticas como aporte para el cuidado enfermero, teniendo como fundamento filosófico la teoría de Jean Watson, y los aportes de Paulo Freire. Métodos: Estudio cualitativo, método descriptivo exploratorio. Participaron 23 adultos con DM2. Escenario de investigación: Consultorio Externo de Endocrinología del H. N. Guillermo Almenara - EsSalud. Los datos fueron recolectados por entrevista abierta a profundidad y se procesaron con el análisis de contenido. Resultados: Los resultados presentaron tres (03) categorías temáticas: Saberes de las personas con DM 2 sobre proceso Salud/ Enfermedad, Prácticas para el (auto) control de la enfermedad y sentimientos; y su relación con los saberes y prácticas. Consideraciones finales: Las personas con DM2 a pesar de saber lo que tienen que hacer para mantener la glucosa normal, presentan patrones conductuales desordenados en su estilo de alimentación, realizan escasa actividad física, e inadecuada adherencia al tratamiento, lo que afecta su estado emocional y determina un inadecuado control de la enfermedad. Los resultados corroboran que el empoderamiento y las acciones que la persona diabética asume con respecto de su enfermedad permiten el no control de la misma. Es esencial que las enfermeras conozcan lo que saben y cómo se cuidan las personas con DM2. Este diálogo permitirá ayudarlos a mejorar su estilo de vida que contribuyen a tener una buena calidad de vida.

Palabras clave: cuidado de enfermería, saberes y prácticas en DM2.

\begin{abstract}
Objectives: Describe the knowledge of the person with diabetes type 2 (DM2) about the disease and the care required. Analyze care practices that person performs according to their knowledge. Discuss the implications of knowing their knowledge and practices as a contribution to nursing care, under the philosophical foundations of the theory of Jean Watson, and the contributions of Paulo Freire. Methods: Qualitative study, exploratory descriptive method. 23 adults with DM2 participated. Research scenario: External Clinic of Endocrinology of Br. N. Guillermo Almenara - EsSalud. Data were collected by open-ended in-depth interview and processed with content analysis. Results: The results presented three (03) thematic categories: Knowledge of people with DM 2 on Health / Disease process, Practices for the (self) control of the disease and feelings; and its relationship with knowledge and practices. Final considerations: People with DM2 despite knowing what they have to do to maintain normal glucose, exhibit behavioral patterns disordered in their eating style, perform little physical activity, and inadequate adherence to treatment, which affects their emotional state and determines an inadequate control of the disease. The results corroborate that the empowerment and the actions that the diabetic person assumes with respect to his illness allow the no control of it. It is essential that nurses know what they know and how people with DM2 are cared for. This dialogue will help them to improve their lifestyle that contribute to having a good quality of life.
\end{abstract}

Keywords: nursing care, knowledge and practices in DM2.

${ }^{1}$ Universidad Norbert Wiener. EAP Enfermería. Lima - Perú.

\section{INTRODUCCIÓN}

El presente estudio surgió de experiencias en el núcleo familiar (adultos mayores) donde varios miembros de mi familia presentaron Diabetes
Mellitus Tipo 2 (DM2), y en el ámbito laboral, como enfermera supervisora noté la frecuencia cada vez más acentuada de personas portadoras de Diabetes Mellitus tipo 2 (DM 2) en los diferentes servicios de hospitalización del Hospital Nacional 
Guillermo Almenara (HNGAI) de EsSalud, los cuales fueron hospitalizados por complicaciones crónicas o agudas de la enfermedad, afectando su calidad de vida; al alta ellos acuden a sus controles médicos periódicos en forma ambulatoria y luego de la consulta son atendidos por la enfermera a cargo, se evidenció que el cuidado que brindan está centrada en la satisfacción de las necesidades biológicas y la administración del tratamiento indicado, como consecuencia de la diversidad de labores encomendadas en su diario quehacer, derivadas de la recarga de trabajo debido al número de pacientes atendidos. También se evidenció una rutinización del trabajo, dejando de lado el cuidado de los aspectos psicológicos, espirituales, sociales y culturales, los cuales conforman al ser humano en su totalidad; olvidando el sentido humanístico de la atención y generando en la persona atendida sentimientos de frustración y descontento.

Esta situación fue corroborada por las entrevistas informales realizadas a las personas con DM2 que asistieron a sus controles médicos programados en la consulta externa, a quienes cuando se les pregunto sobre estilos de vida, la mayoría refirió falta de costumbre de realizar ejercicios y una dieta inadecuada, ya que les era difícil mejorar estas conductas, así mismo indicaron que sintieron que esta situación generó angustia y desesperanza.

Se sabe que la DM2 representa una de las enfermedades crónicas más comunes en el mundo. Algunos organismos de salud la consideran una pandemia creciente (1), por lo que la consideran un problema de salud pública. Así mismo la Federación Mundial de Diabetes (2) afirmó que: Para el año 2030 esta cifra se calcula que aumentará hasta alcanzar los 552 millones de personas con diabetes y otros 398 millones de personas con alto riesgo. Según informes de la Oficina de Estadística e Informática del Ministerio de Salud (3), se reportó que en el Perú, esta enfermedad afecta a casi 2 millones de personas y es la décimo quinta causa de mortalidad, calculando una prevalencia nacional cercana al $8 \%$ y en Lima alrededor del $10 \%$.

Es importante destacar que la DM se ha convertido en una de las mayores dolencias del siglo XXI y una de las más costosas para los sistemas sanitarios. Esto se debe a que un número creciente de personas en el mundo no han tomado conciencia de esta enfermedad (4). Por ello la DM 2 ha sido incluida en la Estrategia Sanitaria Nacional de Prevención y Control de Daños No Transmisibles, establecida desde el 27 de Julio de 2004 con RM N $\mathrm{N}^{\circ} 771-2004 / \mathrm{MINSA}$ teniendo como objetivo principal fortalecer las acciones de prevención y control de los daños no transmisibles, y facilitar la prevención y atención de la salud de las personas, en el marco de la Atención Integral de Salud.

Ante esta situación el Seguro Social del Perú (EsSalud), como parte del Sistema de Salud del país, a través de la Gerencia General, en el marco del Modelo Integral de Salud, decidió fortalecer la atención de salud a la población asegurada, desarrollando el Plan Nacional de Prevención (5). Esta información se corrobora con lo reportado por la Oficina de Gestión y Desarrollo e Inteligencia Sanitaria del HNGAI (6) en sus informes estadísticos del año 2014 al afirmar que la DM2 es la séptima causa de atención en la Consulta Externa, equivalente al $2.41 \%$ de asegurados y de ellos el $1.7 \%$ requirieron hospitalización.

La DM2 es una enfermedad crónica tratable y controlable, pero no curable, que requiere de un tratamiento integral, pero esto sólo puede lograrse a través de un proceso educacional que durará el resto de su vida. La Federación Internacional de Diabetes (7) señala que los diabéticos juegan un papel fundamental en el abordaje de su enfermedad. Por ello, la adecuación para el diabético, es una parte integral de su cuidado. La meta de la educación del diabético es apoyar sus esfuerzos para entender la naturaleza de su enfermedad y tratamiento. En dicha labor interviene todo el equipo de salud, constituyendo un elemento esencial el profesional en enfermería, quien debe buscar alternativas que impulsen acciones o medidas para promover la educación para la salud del diabético, con el objetivo de lograr que la persona sea su propio agente de cuidado. Es por ello que el objetivo del presente estudio está referido a los saberes y prácticas de la persona con DM2, y su implicancia para el cuidado de enfermería.

\section{MATERIALES Y MÉTODOS}

El contexto social donde se realizó el presente estudio fue la Consulta Externa del Servicio de Endocrinología del Hospital Nacional Guillermo Almenara Irigoyen del Seguro Social del Perú (EsSalud), acreditado como Nivel III-2. Los participantes fueron las personas portadoras de DM2 atendidos en esta área, independientemente del sexo. Fueron captados en la sala de espera, desde la primera semana del mes de marzo, a la última semana del mes julio del año 2016.

Para delimitar la muestra se usó la técnica de "saturación", ya que la indagación culminó cuando las entrevistas empezaron a repetirse en su contenido y evaluado el logro de los objetivos de 
la investigación. Se entrevistó un total de 23 personas con DM2 dividido en 17 mujeres y 6 hombres, con 7 entrevistados dentro del grupo etario de adultos (40-60 años) y 16 dentro del grupo de adultos mayores (60 años a mas).

El tipo de investigación del presente estudio es de naturaleza cualitativo, como lo destaca Deobold (11) al referir que el objetivo de la investigación descriptiva consiste en llegar a conocer las situaciones, costumbres y actitudes predominantes a través de la descripción exacta de las actividades, objetos, procesos y personas. $\mathrm{Su}$ meta no se limita a la recolección de datos, sino a la predicción e identificación de las relaciones que existen entre dos o más variables.

El abordaje metodológico fue descriptivo explicativo que parte de lo deductivo a lo inductivo. El estudio descriptivo se dirige fundamentalmente a la descripción de fenómenos sociales o educativos en una circunstancia temporal y especial determinada.

Para la recolección de datos se utilizó la entrevista abierta en profundidad, la cual fue aplicada a las personas con DM2 que posibilitaron y aceptaron la aplicación de la misma, con una duración de 20 a 35 minutos, entre los meses de enero a junio 2016. La entrevista fue abierta y la persona fue invitada a hablar sobre los saberes y prácticas acerca de la DM2. Para delimitar la muestra se hizo uso de la técnica definida como "saturación", que se refiere a que la indagación culminaría cuando las entrevistas empiecen a repetirse en su contenido. Los testimonios fueron grabados con el consentimiento de la participante y se transcribieron tal cual fueron narradas en los discursos posteriormente se realizó el análisis de los relatos, la reducción temática e interpretación de los datos. Los testimonios obtenidos fueron ordenados previo proceso de transliteración en tablas de doble entrada por temas; inicialmente en el lenguaje propio de los informantes o EMIC para luego mediante lecturas sucesivas y reflexión a la luz del referencial teórico, realizar la interpretación en el lenguaje ETIC o del investigador. Las categorías fueron analizadas mediante análisis temático de Bardin (12). A quienes aceptaron participar en el estudio se les solicitó firmar el formulario de consentimiento informado.

El sustento referencial corresponde a los fundamentos filosóficos de Jean Watson, autora de la "Teoría del Cuidado Humano" (8), para conceptualizar el cuidado de enfermería, y para los saberes y prácticas se analiza los aportes de la filosofía de la educación de Paulo Freire $(9,10)$.

\section{RESULTADOS Y DISCUSIÓN}

Las categorías emergieron de las propias narrativas de las personas portadoras de DM2. Fueron construidas a partir de los datos recogidos en las entrevistas, se configuraron las temáticas encontradas y respetó la veracidad de las narrativas (13).

\section{Categoría I. Saberes de las personas con DM 2 en el cotidiano sobre el proceso Salud/ Enfermedad}

Las personas con DM 2 construyen una forma de conocimiento empírico obtenido a lo largo del tiempo que conviven con la enfermedad, en el contexto de vida socio familiar, cultural y político donde viven y a la luz de las estrategias impulsadas por el Sistema Nacional de Salud, experimentando sin embargo las limitaciones que impone la enfermedad como consecuencia de los estilos de vida llevados en el tiempo. Puede tratarse de un saber teórico, saber hacer, reflexivo, saber actuar, según el contexto en el que construye el saber (13). A través de lo expresado por los participantes, se agruparon las ideas y conceptos similares configurando las siguientes sub categorías

\section{Sub categorías:}

- Saberes centrados en la experiencia práctica de la persona con diabetes:

Es de suma importancia evaluar los métodos de enseñanza que se imparten a las personas diabéticas, e investigar que tan interesado está la persona en su enfermedad y elaborar un perfil de su esfera psicosocial, pues se sabe que sobre el control la persona diabética intervienen algunas variables de ésta esfera, que se correlacionan con las fluctuaciones de la glicemia $(14,15)$.

La metodología cuestionadora (16) de Freire se orienta por la percepción de la realidad, por el protagonismo y por el trabajo en grupo. El aprendizaje debe estar asociado a la tomada de conciencia de una situación real vivida por lo educando, en este caso, el paciente. Para Freire, enseñar no es transferir conocimientos, pero crear las posibilidades para su construcción $(17,18)$.

Según Watson, el profesional debe adoptar una conciencia de cuidado genuino, compartir información para promover el conocimiento y ampliar las posibilidades de las personas para construir formas de cuidar de sí mismas (8).

\section{- Atribución de la enfermedad a eventos de la vida cotidiana.}

$\mathrm{Al}$ respecto Freire (10), refiere que se aprende con reciprocidad de conciencias. El educador es un coordinador que tiene por función dar, las 
informaciones solicitadas por los respectivos participantes y propiciar condiciones favorables a la dinámica de grupo. Lo que el hombre habla y escribe y como habla y escribe, es toda expresión objetiva con su espíritu.

Para el desarrollo de la educación para la salud es esencial conocer el estilo de vida, las creencias y los valores de los pacientes y desde allí pensar en las prácticas de atención. Sin acceso a este conocimiento, de hecho la actividad educativa no logrará el éxito esperado.

- Conocimientos sobre las manifestaciones clínicas de la enfermedad y sus complicaciones.

En general tienen una idea clara de las complicaciones de la DM2, aunque las perciben como algo que poco o nada pueden modificar. Hoy en día, el autocontrol de la diabetes es parte integral y crítica del plan terapéutico de las personas con diabetes.

La filosofía de la educación según Freire, parte de la concepción de la acción humana como un acto reflexivo, por el cual el pensamiento adquiere valor en la acción. En la interacción con la persona es indispensable pasar de la contemplación ingenua de la enfermedad y sus efectos, a la criticidad del papel que juega el individuo y su contexto político y sociocultural en su salud (10).

\section{Categoría II. Prácticas para el (auto) control de la enfermedad.}

Las personas con DM2 están conscientes de que una de las causas más importantes de la falta de control de la enfermedad es la constante transgresión dietética o falta de apego al tratamiento, como si existiera un duelo no resuelto debido a la pérdida de la salud.

\section{Sub categorías:}

\section{- Hábitos alimentarios.}

Mayeya (19), enfatiza la gran dificultad que representa el cambio de hábitos alimenticios de la infancia, tanto en la preparación como en el consumo de alimentos y la progresiva disminución de los alimentos prohibidos. Las categorías mostradas guardan similitud con los resultados de García Reza (20), en el sentido de revelar la existencia de una dolencia que compromete de modo holístico la salud de las personas, es multicausal y genera sentimientos propios y en contextos extremadamente limitantes si progresan las complicaciones.

En la misma dirección, Freire (18) señala que existe la confianza cuando hay coherencia entre las palabras y acciones: "Para decir una cosa y hacer otra, no tomar en serio la palabra, no puede ser estimulante para la confianza". En este sentido, las limitaciones de los sujetos en el cumplimiento de la dieta no debe ser motivo de un juicio de los profesionales sanitarios. Pero debe entenderse como una situación compleja, que implica rupturas y el estigma social, así como las cuestiones culturales fuertes inherentes al contexto de la vida sujeto.

\section{- Tipos de actividades físicas}

Todos sin duda atribuyen efectos benéficos al ejercicio, sin embargo no tienen una idea clara respecto a los beneficios de éste en la hiperglicemia. Por otra parte, no lo consideran como parte del tratamiento de la DM2.

En relación a las prácticas realizadas por las personas con DM2, Watson (8) indica que la necesidad de ingerir alimentos y ejercicios es mucho más que una demanda de supervivencia, por estar relacionados con cuestiones culturales, con significados y bienes simbólicos, asociados con sentimientos de amor, amistad, la confianza y la seguridad en las relaciones humanas. Por lo tanto, las enfermeras y el personal médico, necesitan modificar su enfoque en la asistencia del paciente: no se trata de una mirada instrumental y fragmentada de los cuidados a observar en el hogar; se trata de comprender que la modificación de hábitos antiguos y la superación de los obstáculos para llevar de modo regular una actividad física, requieren una interacción educativa que es el tratamiento del paciente, el intercambio constructivo de información que prepare a la persona para la denominada educación terapéutica, es decir una formación de la persona para la autogestión de los cambios requeridos en las diferentes esferas de su vida, para adaptarse y realizar sus proyectos con calidad de vida (20).

Categoría III. Expresión de sentimientos y su relación con saberes y prácticas de personas con DM 2.

Se evidencia una frustración debida al impacto que la enfermedad ha tenido dentro de sus rutinas de vida, ya que se sienten que se ven privados de actividades que antes realizaban con normalidad, pero ahora requieren consideraciones adicionales. García Reza (20), coincidentemente con los resultados descritos, incluye la tristeza, desesperación, miedo, deseo de llorar y llegar al límite de no querer vivir más. La comprensión en términos de Watson (8), que la expresión de las emociones son ventanas del alma del paciente, implica uno de los atributos del cuidado que emergen en este estudio: conocer lo que la persona experimenta para desde ese espacio, construir un encuentro empático y en esencia humanizante. 


\section{CONSIDERACIONES FINALES}

Las personas con DM2 a pesar de saber lo que tienen que hacer para mantener la glucosa normal presentan patrones conductuales desordenados en su estilo de alimentación, no realizan actividad física, falta de adherencia al tratamiento, lo que determina un inadecuado control de la enfermedad y afecta su estado emocional. Los resultados corroboran que el empoderamiento y las acciones que la persona con diabetes asume al respecto de su enfermedad permiten el control de la enfermedad. Es esencial que las enfermeras conozcan lo que saben y cómo se cuidan las personas con DM2. El dialogo con ellos permite cambios para ayudar a mejorar su estilo de vida que contribuyen a tener una buena calidad de vida.

\section{AUTOR DE CORRESPONDENCIA}

Gaby Sonia Chávez Zegarra

EAP de Enfermería

Universidad Norbert Wiener

E-mail: gchavezz@hotmail.com

Teléfono: 98576350

\section{REFERENCIAS BIBLIOGRÁFICAS}

1. (OMS-WHOQOL) OM de la S. Health Promotion Glossary [Internet]. Géneva. 1998 [cited 2013 Sep 3]. p. 17. Available from: http://www.who.int/hpr/NPH/docs/hp_glossar y_en.pdf

2. Federación internacional de la Diabetes. Plan Mundial contra la diabetes 2012-2021 [Internet]. p. 4. Available from: http://www.idf.org/sites/default/files/attachme nts/GDP-Spanish.pdf

3. MINSA. Oficina de Estadística e Informática del Ministerio de Salud [Internet]. Especial de diabetes. 2010. Available from: http://www.minsa.gob.pe/portada/Especiales/ 2010/diabetes/datos.asp

4. Amezcua Martínez M. Antropología de los Cuidados enfermedad y padecimiento: significados del enfermar para la práctica de los cuidados. Cult los Cuid. 2000;IV(7-8):607.

5. ESSALUD. Plan Nacional de Prevención de EsSalud 2012. RGG N ${ }^{\circ}$ 372- GG- ESSALUD. 2012;4.

6. Hospital Guillermo Almenara Irigoyen de ESSALUD. OGID: Reporte del perfil epidemiológico semestral, Lima Perú. 2014
7. Federación Internacional de Diabetes. Guía para diabético tipo 2 [Internet]. Clinical Guidelines Task Force. 2005 [cited 2014 Jun 5]. Available from: www.idf.org/home/indez.cfm?node $=1549$

8. Watson J. Nursing: the Philosophy and Science of caring. Boston: Little Brown and Company; 1979. 32

9. Freire P. Educação como prática de liberdade. Rio de Janeiro: Paz e Terra; 1967.

10. Freire P. Pedagogia do oprimido. Rio de Janeiro. Rio de Janeiro: Paz e Terra; 1975.

11. Van Dalen DB, Meyer WJ. Manual de técnica de la investigación educacional [Internet]. Paidos Iberica; 1981. Available from: http://noemagico.blogia.com/2006/091301-lainvestigacion-

12. Bardin L. Análisis de contenido. 70th ed. Brasil: Ediciones AKAL; 2011.

13. Minayo M, Ferreira Deslandes S, Cruz Neto O, Gomes R. Investigación social: teoría, método y creatividad. 3rd ed. Petrópolis: Editora Vozes; 1994. 80 p.

14. Araúz A, Sánchez G, Padilla G, Fernández M, Rosello M, Guzmán S. Intervención educativa comunitaria sobre diabetes en el ámbito atención primaria. Rev Panam Salud Púbica. 2001;9:145-69.

15. García G, Suárez P, Acosta O. Comunicación y educación interactiva en salud y su aplicación al control del paciente diabético. Rev Panam Salud Púbica. 1997;2:12-7.

16. Bordenave J, Pereira A. Estratégia de ensino aprendizagem. 24th ed. Petrópolis: Vozes; 2002. $312 \mathrm{p}$.

17.Freire P. Pedagogia da autonomia: saberes necessários à prática educativa. 25 th ed. Rio de Janeiro: Paz

18. Freire P. Conscientização: teoria e prática da libertação. Uma introdução ao pensamento de Paulo Freire. 3rd ed. São Paulo: Moraes; 1980. $87 \mathrm{p}$.

19. Mayeya PP. Conocimiento sobre diabetes y conductas alimentarias en pacientes con diabetes tipo II en atención ambulatoria del servicio de endocrinología, hospital Guillermo Almenara Irigoyen. Universidad Ricardo Palma, Perú; 2011.

20. García-Reza C, Alvirde-Vara R, LanderosLópez M, Solano-Solano G, Medina-Castro M. Resistencia frente a la enfermedad: relato de un cuerpo dañado por la diabetes [Internet]. Comunidad de Santa Cruz México. Aquichan, Norteamérica, 2009-2010. [cited 2014 Jan 14]. Available from: http://aquichan.unisabana.edu.co/index.php/a quichan/article/view/3082. 